Article

\title{
Primary Control Method of Wireless Charging System Based on Load Characteristics
}

\author{
Guodong Chen ${ }^{1,2}$, Chao Rao ${ }^{1}$, Yue Sun ${ }^{1, *}$, Zhenxin Chen ${ }^{2}$, Chunsen Tang ${ }^{1}$ and \\ Zhenpeng Zhang ${ }^{3}$ \\ 1 College of Automation, Chongqing University, Shapingba District, Chongqing 400030, China; \\ 20121301011@cqu.edu.cn (G.C.); 20161313015 t@cqu.edu.cn (C.R.); cstang@cqu.edu.cn (C.T.) \\ 2 State Grid Zhejiang Electric Company Zhoushan Power Supply Company, Zhoushan 316000, China; \\ tangbo@shiep.edu.cn \\ 3 China Electric Power Research Institute, Wuhan 430074, China; zhangzhenpeng@epri.sgcc.com.cn \\ * Correspondence: syue@cqu.edu.cn; Tel.: +86-135-0836-8896
}

Received: 24 February 2019; Accepted: 28 March 2019; Published: 2 April 2019

\begin{abstract}
Aiming at the output control issues of a lithium ion battery wireless charging system, a primary side control method based on load characteristic identification is proposed. The primary side impedance is calculated by detecting the effective value of the primary side voltage and current, and the mapping relationship between the equivalent load and the primary side impedance is established based on the AC impedance model. Using this mapping relation, the output of the secondary side can be regulated indirectly by controlling the input voltage of the inverter. Compared with the traditional control methods, the proposed control method not only eliminates the communication requirement between the primary side and secondary side, but also simplifies the hardware circuit design, reduces the complexity of the control circuit and also reduces the volume and cost of the system. In the paper, the impedance characteristics of the lithium ion battery at constant current and constant voltage stage are analyzed. The principle of the primary side control method is expounded and the realization method is given. The feasibility of the proposed control method is verified by simulation and experiment.
\end{abstract}

Keywords: inductively coupled power transfer; wireless charging; load identification; primary side control

\section{Introduction}

Inductive power transfer (IPT) technology employs a high-frequency magnetic field to construct an energy transmission channel between power supply equipment and electrical equipment, realizing power transmission in a non-contact manner [1-5] and overcoming the disadvantages of traditional contact power supply under harsh environments (such as high humidity, high temperature, high corrosion, and explosive environment). It is characterized by high reliability, high safety and low maintenance [6-10], and has been widely employed in electric vehicles [11], underwater robot [12] and other fields.

In IPT system applications, the output voltage or output current is typically controlled to meet different load requirements. At present, the commonly utilized control methods of IPT systems can be generally summarized as the direct control of the primary side, cooperative control of the primary and secondary side, and indirect control of the primary side.

The direct control method of the primary side detects the output voltage and current information in real time on the secondary side and then sends the detection information to the primary side through wireless communication. The primary side controller performs corresponding control based 
on the received voltage and current information to achieve direct control of output [13]. The wireless communication methods commonly used in IPT systems, including the radio frequency, bluetooth, zigbee and synchronous transmission of energy signals [14-17] and so on. Due to the direct detection and control of the output, it can achieve precise control. However, this method also has some problems. Firstly, the transmission of information by wireless communication will bring the delay of feedback information, affecting the control speed and even security performance of the system, as well as increasing the cost. Second, in some special occasions, such as the underwater environment, the electromagnetic environment of the system is complicated, resulting in high error rate, low baud rate and other problems of wireless communication.

The cooperative control method of the primary and secondary side involves control modules in both sides. Usually, the secondary side control module controls the output quantity by collecting the output voltage and current information, and the primary side controller performs the power control according to the output requirement. The literature [18] proposed a scheme to achieve constant voltage and current control of the output of secondary voltage by adding a controllable protection circuit on the secondary side to limit the output voltage and current while performing power control on the primary side. This method has the advantages of fast control speed and high control precision. However, in order to achieve constant voltage and current control, a controllable protection circuit is added, which increases the size and cost of the system. In Reference [19], a dynamic tuning circuit is added on the secondary side to control the output power by changing the resonance degree of the secondary side. This method can make the operating frequency of the system consistent with the natural resonance frequency and has better dynamic performance but greatly increases the control complexity of the system. The literature $[20,21]$ proposed a method to achieve maximum efficiency tracking by adding DC-DC converters to the primary and secondary sides to control the input and output power respectively.

For the indirect control method of the primary side, in which the detection and control are executed on the primary side, by establishing the mapping relationship between the output of the secondary side and relevant quantities of primary side, such as voltage and current, the indirect control of the output is realized without the need for the closed loop control of the primary side and secondary side. At present, there are many research literatures on the identification of loads by detecting relevant information on the primary side [22-24]. However, these literatures mainly focus on load identification of different topologies. Besides load identification, there is no further research on power control methods. In Reference [25], a primary side control scheme is proposed by collecting the compensation capacitor voltage and inverter current of the primary side and then performing signal processing to obtain the phase difference. The corresponding output information of the secondary side was calculated and then compared with the set value to obtain the error information. On the basis of the error information, the corresponding control method was required. The scheme effectively overcomes the communication requirements of the primary and secondary sides, but it should obtain the phase difference information of the voltage and current of the primary side; this increases the complexity of the detection circuit and the program processing. In addition, the detection accuracy of the phase difference directly affects the control accuracy of the system. In Reference [26], a parameter identification and primary side control method were proposed. However, on the one hand, this method requires adding two more resonant coils between the primary and secondary coils, which increases the system volume and cost. On the other hand, this method can only realize the constant voltage output of the secondary side and lack of research on the constant current control.

In general, the three kinds of control methods have their own advantages and disadvantages. In this paper, a primary side indirect control method based on load characteristic identification is proposed for the lithium ion battery wireless charging system, without the need for a wireless communication channel and additional hardware circuit. By detecting the primary side inverter output current and voltage and controlling the primary side impedance, the output voltage and current of the secondary side can be controlled. Compared with the existing primary side indirect control method, it has the 
advantages of high precision, easy implementation, low cost and small size. This paper aims at the SS-compensated lithium-ion battery wireless charging system, based on the analysis of the power transfer characteristics of constant current and constant voltage floating charge of the lithium-ion battery. The principle of the primary-side control strategy in the constant current and constant voltage phase is given. And the implementation shows that the feasibility of the proposed control method is verified by simulation and experiment.

\section{Materials and Methods}

\subsection{Wireless Charging System}

In this paper, the wireless charging system topology of lithium ion battery is shown in Figure 1. In order to control the transmission power, a DC/DC converter is added between the power supply and the inverter bridge. $E_{d c}$ is the DC input voltage of the system, $L_{p}$ and $L_{s}$ are the self-inductance of the transmitting coil and the receiving coil respectively, $C_{p}$ and $C_{s}$ are corresponding thereto. $R_{p}$ and $R_{s}$ are the corresponding equivalent series resistance, and $\mathrm{M}$ is its mutual inductance. $E_{d c}$ performs voltage regulation through a DC/DC converter and is inverted into a square wave voltage through the switching network S1 S4 and drives the primary side resonant network to generate an alternating current on the energy transmitting coil $L_{p}$ to excite the high frequency magnetic field. The power picked up by the secondary side receiving coil is compensated, rectified, and filtered to charge the battery, and diode $D_{2}$ is used to prevent the current from flowing backwards. $U_{0}$ is the charging voltage and $I_{O}$ is the charging current. For the convenience of analysis, the battery model is equivalent to impedance load $R_{\text {eq }}$.

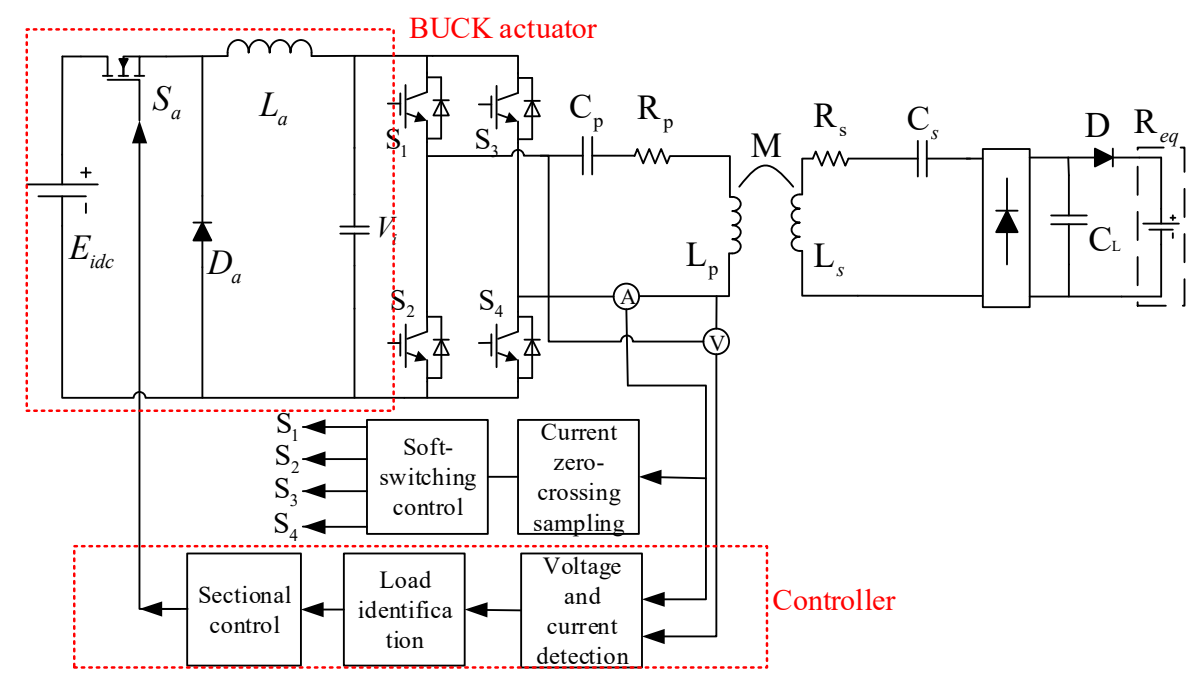

Figure 1. Diagram of SS type lithium ion battery wireless charging system.

In the charging process, in order to improve the transmission efficiency of the system, the zero-crossing detection of the primary resonant coil current is performed, and the driving signal of the inverter switching tube is generated according to the zero-crossing signal. To prevent the output voltage oscillation of the inverter, setting up the primary side resonance network is weakly inductive so that the inverter link operates in the zero voltage switch (ZVS) soft switching state. At the same time, by collecting the inverter output voltage and current to identify the load characteristics of the output, the identification result is compared with the reference value to obtain the error signal, and then the corresponding control is implemented to realize the constant current and constant voltage charging of the lithium ion battery at the output terminal. 


\subsection{Load Characteristic Analysis}

\subsubsection{Analysis of Impedance Characteristics of Batteries at Different Charging Stages}

Commonly used lithium-ion batteries are generally charged in a three-stage charging mode. (1) Pre-charging, when the battery voltage is less than the minimum threshold voltage, a small current is used to pre-charge the battery to raise the battery voltage to the threshold voltage. (2) Constant current charging, at which the charging current is kept constant and the charging voltage rises slowly. (3) Constant voltage charging, in which the charging voltage is remained constant and the charging current is rapidly decreased [27]. In general, battery over-discharge or over-charging has a great impact on battery life. Therefore, for lithium-ion batteries, the charging process has two main stages, namely constant current stage and constant voltage stage. The formula for calculating the equivalent impedance of the battery is as follows:

$$
R_{e q}=\frac{U_{O}}{I_{O}}
$$

The charging process curve and impedance characteristic curve are shown in Figure 2. It can be seen that during the whole charging process, the equivalent impedance of the battery shows a trend of gradual increase. In the constant current stage, due to slow voltage rise, the trend of impedance increase is relatively gentle, while in the constant voltage stage, due to rapid current decline, the trend of impedance increase is relatively steep.

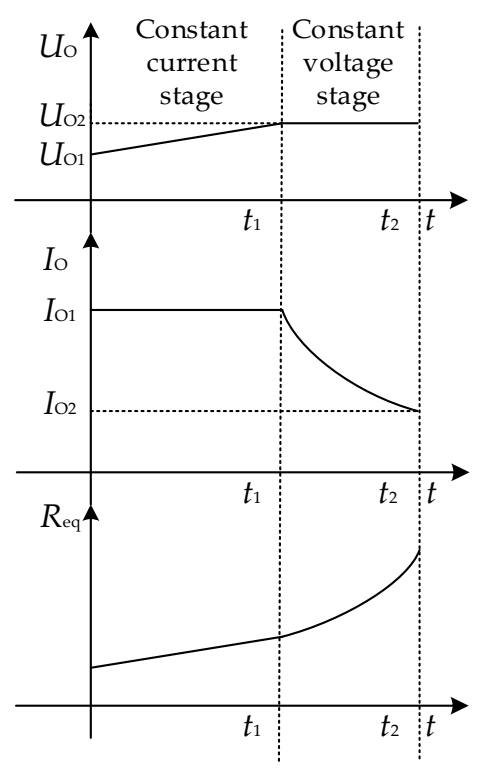

Figure 2. Charging characteristic curve of battery.

\subsubsection{System Load Characteristic Model}

For the system topology which is shown in Figure 1, the secondary side equivalent circuit is shown in Figure 3.

In the Figure, $j \omega M I_{p}$ is the induced voltage of the secondary receiving coil, and $I_{p}$ is the inverted output current of the primary side. In order to make the system be in the resonance state, the resonant angular frequency is designed as $\omega^{2}=L_{S} C_{S}$, which is further simplified to the circuit shown in Figure 4. 


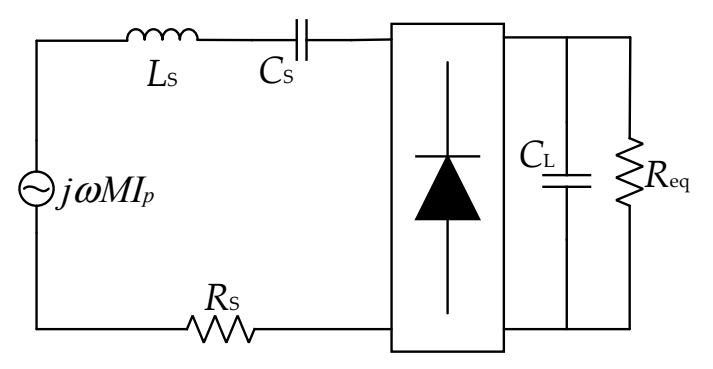

Figure 3. Equivalent circuit diagram of the secondary side.

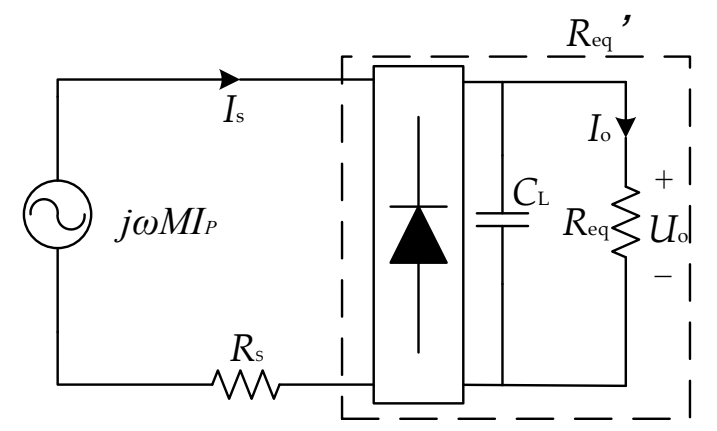

Figure 4. Simplified equivalent circuit of secondary side.

As it is shown in Figure 4, according to the law of conservation of energy, the total equivalent impedance of the rectifier bridge and the battery load is:

$$
R_{e q}{ }^{\prime}=\frac{8}{\pi^{2}} R_{e q}
$$

The total impedance of the secondary side is:

$$
Z_{S}=R_{S}+R_{e q}^{\prime}
$$

The impedance reflected to the primary side is:

$$
R_{Z}=\frac{\omega^{2} \mathrm{M}^{2}}{Z_{S}}=\frac{\omega^{2} \mathrm{M}^{2}}{R_{S}+R_{e q}^{\prime}}
$$

Therefore, the equivalent circuit of the primary side is shown in Figure 5:

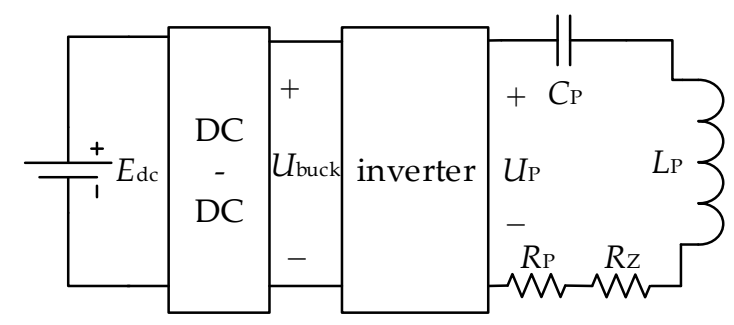

Figure 5. Equivalent circuit diagram of primary side.

Under the resonance state, the total impedance of the primary side can be expressed as:

$$
Z_{P}=R_{P}+R_{Z}=R_{P}+\frac{\omega^{2} M^{2}}{R_{S}+R_{e q}}
$$


In accordance with Equation (5), it can be seen that the equivalent load $R_{e q}{ }^{\prime}$ has a one-to-one correspondence with the primary side impedance $Z_{P}$. The DC-DC module uses BUCK circuit, so the primary side DC input has the following relationship with the inverted output:

$$
U_{P}=E_{d c} \times D \times \frac{4}{\sqrt{2} \pi}=\frac{4}{\sqrt{2} \pi} U_{b u c k}
$$

where, $\mathrm{D}$ is the duty cycle of the BUCK circuit and $U_{P}$ is the inverter output voltage. Through the analysis of the primary and secondary sides, the entire system can be simplified and the circuit is shown in Figure 6.

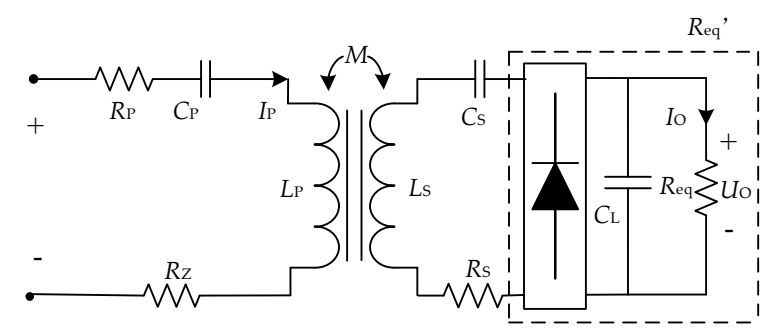

Figure 6. Equivalent circuit diagram of both sides.

As it is shown in Figure 6, the primary current can be obtained as:

$$
I_{P}=\frac{U_{P}}{Z_{P}}=\frac{U_{P}\left(R_{S}+R_{e q}{ }^{\prime}\right)}{\omega^{2} M^{2}+R_{P}\left(R_{S}+R_{e q}{ }^{\prime}\right)}
$$

The pickup voltage of the secondary side is:

$$
U_{S}=j \omega M I_{p}=j \frac{\omega M U_{P}\left(\mathrm{RSR}_{S}+R_{e q}{ }^{\prime}\right)}{\omega^{2} M^{2}+R_{P}\left(R_{S}+R_{e q}{ }^{\prime}\right)}
$$

As shown in Figure 4, the average value after rectification is:

$$
U_{O}=\frac{\pi}{2 \sqrt{2}} \frac{R_{e q}^{\prime}}{R_{e q}{ }^{\prime}+R_{S}} \omega M I_{p}
$$

On the basis of Equations (6)-(9), the output voltage of the secondary side is:

$$
U_{O}=\frac{\omega M R_{e q}{ }^{\prime}}{\omega^{2} M^{2}+R_{P}\left(R_{S}+R_{e q}{ }^{\prime}\right)} U_{b u c k}
$$

The output current of the secondary side is:

$$
I_{O}=\frac{8}{\pi^{2}} \frac{\omega M}{\omega^{2} M^{2}+R_{P}\left(R_{S}+R_{e q}{ }^{\prime}\right)} U_{b u c k}
$$

From Equation (5), the relationship between the primary side impedance and the output of the secondary side can be obtained as follows:

$$
\begin{gathered}
U_{O}=\frac{\omega^{2} M^{2}-Z_{p} R_{s}+R_{p} R_{s}}{\omega M Z_{p}} U_{b u c k} \\
I_{O}=\frac{8}{\pi^{2}} \frac{Z_{p}-R_{p}}{\omega M Z_{p}} U_{b u c k}
\end{gathered}
$$


In a specific system, $\omega, M, R_{P}, R_{S}$ are known. Basing on Equation (7), as long as the inverter output voltage $U_{P}$ and the inverter current $I_{p}$ are measured at the primary side, the primary side impedance $Z_{p}$ and a corresponding equivalent impedance $R_{e q}{ }^{\prime}$ can be obtained, and then the secondary side output voltage and output current can be obtained by Equations (12) and (13). The deformation of the Formula (12) and (13) can be expressed as:

$$
\begin{gathered}
Z_{p}=\frac{\omega^{2} M^{2}+R_{p}, R_{s}}{\omega M U_{o}+R_{s} U_{b u c k}} \\
Z_{p}=\frac{8 R_{p}}{8 U_{\text {buck }}-\pi^{2} \omega M I_{o}} U_{\text {buck }}
\end{gathered}
$$

Therefore, by adjusting the output voltage of BUCK, the primary impedance can be adjusted to control the output voltage $U_{o}$ and the output current $I_{o}$.

\subsection{Primary Indirect Control Strategy}

The control scheme proposed in this paper is to detect and control at the primary side directly by using the corresponding relationship between the primary side impedance and the secondary side output voltage and current. According to Equation (5), there is a one-to-one correspondence between the primary side impedance and the secondary side impedance. As the charging process proceeds, the correspondence relationship between the equivalent impedance of the battery, the primary side impedance, and the battery power (SOC) are as shown in Figure 7. For the convenience of analysis, the inherent impedance characteristic curve of the lithium ion battery is represented as $R_{\text {eqref }}$. Similarly, the corresponding primary equivalent impedance is $Z_{\text {pref }}$.

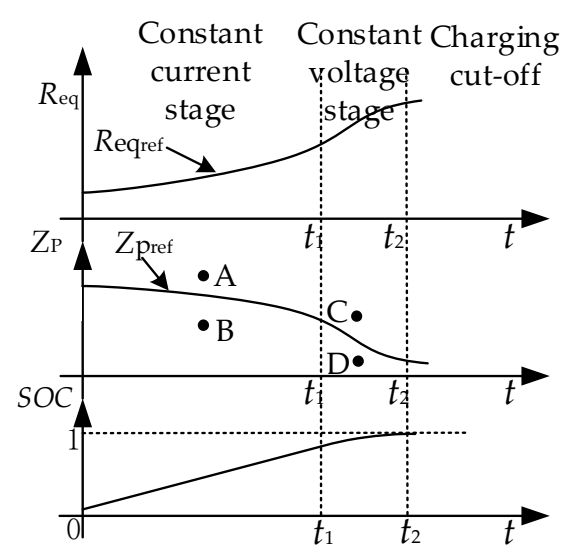

Figure 7. The diagram of both side impedance and SOC of battery.

During the charging process, the output voltage and current of the secondary side need to be controlled. Basing on Equation (1) and Figure 7, these two variables can be expressed by the equivalent impedance of the battery. Since Figure 7 is derived based on Figure 2 and combined with the above relevant formulas, it is known that the charging voltage and current are the same as those at the same time in Figure 2 when the $Z_{p}$ is controlled as $Z_{\text {pref }}$. In other words, during the whole charging process, constant current and constant voltage can be achieved by controlling $Z_{p}$ to follow the $Z_{\text {pref }}$ curve.

In the charging process of a lithium ion battery, the first stage is a constant current charging, which accounts for the majority of the whole charging process. In this stage, the voltage of battery rises slowly. In each control cycle, the voltage of battery is approximately unchanged. In the light of Equations (7) and (12), the charging voltage can be calculated, and $Z_{p}$ can be adjusted to follow the 
$Z_{\text {pref }}$ curve by adjusting the BUCK output voltage due to Equation (14). From Equations (1), (5) and (8), the following relations can be obtained:

$$
Z_{P} \uparrow \leftrightarrow \frac{U_{P}}{I_{P}} \uparrow \leftrightarrow R_{\text {eq }} \downarrow \leftrightarrow \frac{U_{O}}{I_{O}} \downarrow
$$

The impedance value of the primary side can be obtained by detecting $U_{p}$ and $I_{p}$. If it is consistent with the reference value $Z_{\text {pref }}$, the control will be maintained. If the primary side impedance value is detected at point $\mathrm{A}$, and it is greater than $Z_{\text {pref, }}$, since $U_{O}$ is approximately constant in a very short time, then according to Equation (16), $\frac{U_{O}}{I_{O}}<R_{\text {eqref }}$, which is caused by the large $I_{O}$, combined with Equation (13), it is known that the $U_{b u c k}$ is required to reduce at this time, and the duty cycle of the BUCK circuit is decreased until point A returns to the primary side impedance curve when the input voltage remains unchanged. Similarly, when it is detected that the primary side impedance value appears at point $B$, the duty cycle of the BUCK circuit needs to be increased.

During the constant voltage charging stage, the equivalent impedance of the battery will rise rapidly due to the decrease of the charging current, and the primary side impedance will continue to decrease. Similarly, the primary side impedance value can be obtained by detecting the $U_{P}$ and $I_{P}$. If it is consistent with the reference value, the duty cycle will remain unchanged. If the primary side impedance value is detected at point $C$ and greater than the reference value, the duty cycle of the BUCK circuit should be reduced based on Equations (9) and (16). Similarly, it is necessary to increase the duty cycle at point D. The control flow of the whole charging process is shown in Figure 8.

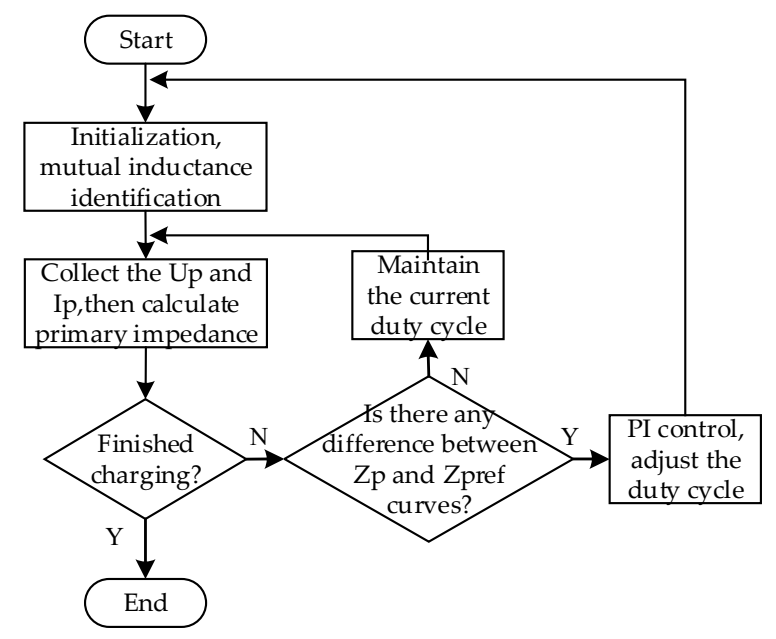

Figure 8. The flow chart of control.

In practical applications, the equivalent impedance and primary impedance of the battery will be different because of the different initial charge quantities, that is, the starting point of the impedance reference curve is uncertain. If there is communication between the primary and secondary sides, the battery-related information can be sent to the primary side as reference value. In the absence of communication links, according to the basic idea of primary side impedance control, this paper proposes a simplified method of constant resistance control for this problem. In accordance with the literature [28], the maximum voltage range of a single lithium-ion battery is $2.75 \mathrm{~V}-4.2 \mathrm{~V}$ during the whole charging period, while the normal voltage range of a single lithium-ion battery is $3.1 \mathrm{~V}-4.1 \mathrm{~V}$ for high-power electrical equipment such as electric vehicles. In order to ensure the service life of the battery, the maximum voltage variation range is $3.5 \mathrm{~V}-4.1 \mathrm{~V}$ in the constant current charging stage [29], that is, the voltage changes just a little. On the basis of Formula (1), the equivalent impedance of the battery changes little in the constant current stage. Therefore, the constant impedance control at the primary side in the constant current charging stage can also get the approximate effect, and because the control impedance is constant, the problem of the different initial power of the battery is also solved. 


\section{Results}

\subsection{Simulation Analysis}

In order to verify the effectiveness of the primary-side impedance control scheme, the whole charging process is simulated and verified according to the topology which is shown in Figure 1. Firstly, the charging characteristic curve of the battery is obtained by the traditional primary side direct control scheme, and the primary side impedance variation curve under the control scheme is obtained by the correspondence relationship between the primary and secondary side impedances. Finally, taking the primary side impedance curve as a reference, the voltage $U_{p}$ and current $I_{p}$ of the primary side are collected in real time and converted into impedance, which is compared with the previously obtained primary side impedance curve. The error is taken as the control basis to obtain the charging characteristic curve of the battery under the primary side impedance control scheme.

For parameter design methods, there are relevant literature researches [5]. However, due to the existence of certain errors between the actual values of the components and the calibration values, in order to make the simulation and experimental results more comparable, the simulation parameters in this paper are consistent with the experimental parameters, which are shown in Table 1.

Table 1. Values of system.

\begin{tabular}{cc}
\hline IPT System Parameters & The Parameter Value \\
\hline Input dc voltage $/ V_{d}(\mathrm{~V})$ & 300.00 \\
Primary resonance capacitance $/ C_{p}(n \mathrm{~F})$ & 13.88 \\
Primary coil self-inductance $/ L_{p}(\mu \mathrm{H})$ & 253.40 \\
Primary coil internal resistance $/ R_{p}(\Omega)$ & 0.43 \\
Auxiliary resonant capacitance $/ C_{p}(n \mathrm{~F})$ & 14.40 \\
Secondary coil self-induction $/ L_{p}(\mu \mathrm{H})$ & 246.00 \\
Secondary side coil internal resistance $/ R_{p}(\Omega)$ & 0.44 \\
Resonance natural frequency $/ f(\mathrm{kHz})$ & 85.00 \\
Rated current $/ I(\mathrm{~A})$ & 8 \\
Rated power $/ P(\mathrm{~kW})$ & 2 \\
\hline
\end{tabular}

Under the primary direct control scheme, the resulting waveform is shown in Figure 9.
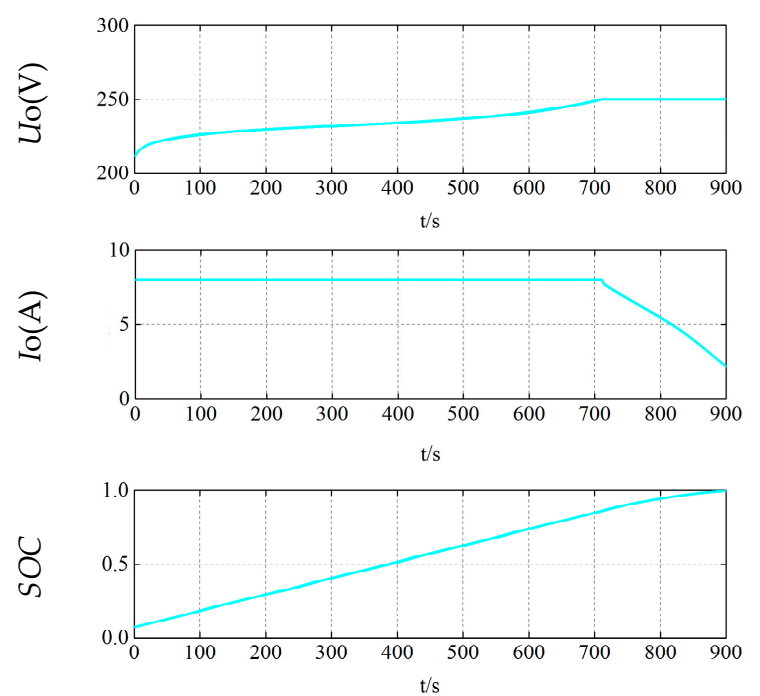

Figure 9. Charging characteristic curve of battery under primary side control.

As can be seen from Figure 9, in the constant current stage, the charging current of the battery is $8 \mathrm{Amp}$, and the battery voltage rises slowly until $250 \mathrm{~V}$. Then it enters the constant voltage mode; 
from the SOC of the battery, we can see that in the constant current stage, the power of the battery has reached about $80 \%$. In the constant voltage stage, the current drops rapidly and the battery is filled quickly.

Similarly, the equivalent impedance curve of the battery and the impedance curve reflected to the primary side are shown in Figure 10.
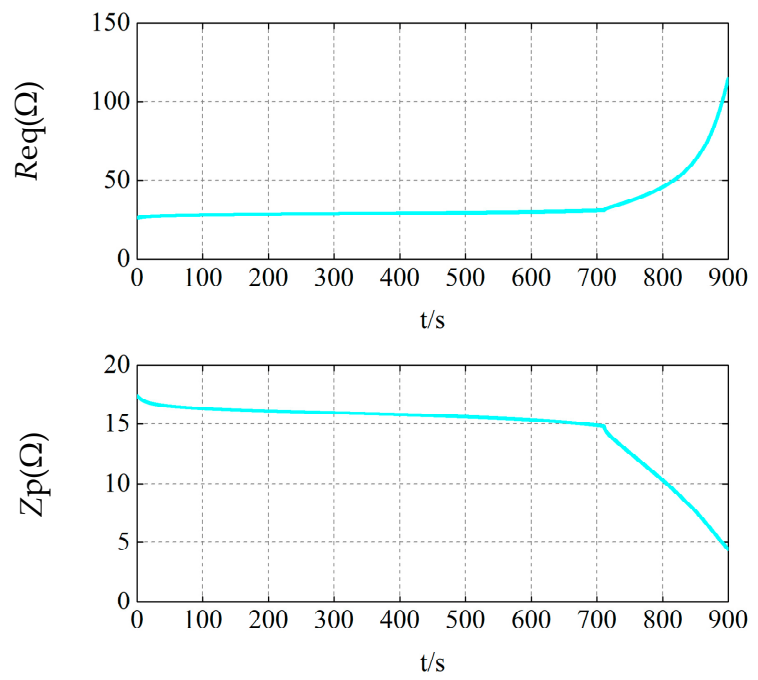

Figure 10. The curve of equivalent load and primary side impedance.

It can be seen from Figure 10, in the constant current stage, the equivalent impedance of the battery increases slightly, almost remains unchanged, and the primary impedance decreases slightly. However, in the constant voltage stage, the equivalent impedance of the battery changes greatly and increases rapidly, while the primary impedance decreases rapidly.

Since each impedance value uniquely corresponds to the state of charge of one battery, the same effect can be achieved by controlling the primary impedance as shown in Figure 10.

In the primary side impedance control scheme, the primary side impedance curve obtained above is used as a reference. The primary side voltage and current are collected in real time, so the primary side impedance can be calculated. Then the error value is obtained by comparing with the reference impedance curve, and the duty cycle of the BUCK circuit is adjusted based on its error. The control effect is shown in Figure 11.

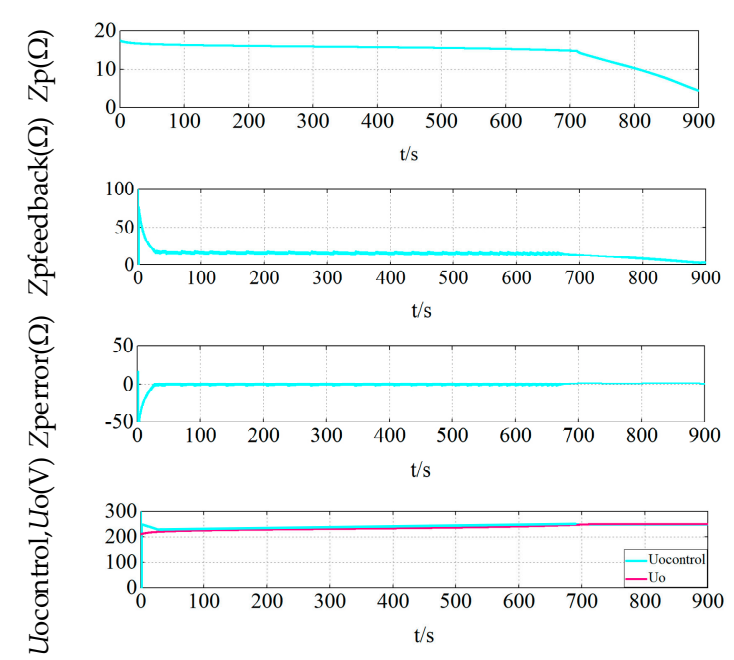

Figure 11. The diagram of closed loop control. 
From Figure 11, taking the $Z_{p}$ as a reference curve, the primary side impedance curve $Z_{p f e e d b a c k}$ is collected in real time, and the error value $Z_{\text {perror }}$ is approached to zero by incremental PI control. Finally, the output voltage curve $U_{\text {ocontrol }}$ obtained under closed-loop control is substantially coincident with the output voltage curve $U_{o}$ directly controlled by the primary side. The battery charging characteristic curve under closed loop control is shown in Figure 12.

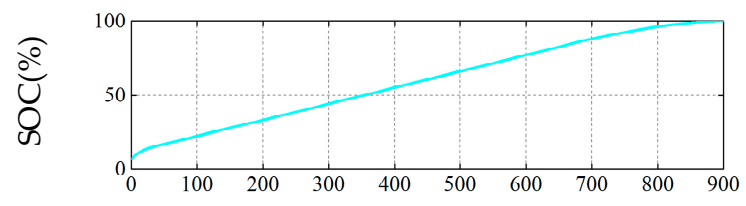

$\mathrm{t} / \mathrm{s}$
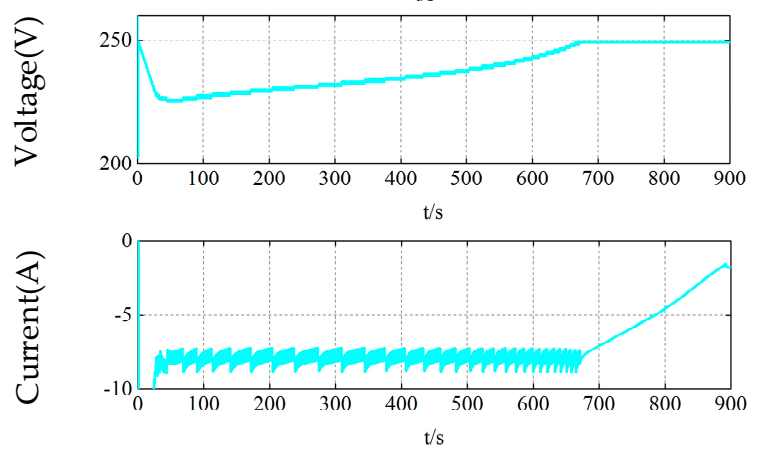

Figure 12. The characteristic curve of the primary side impedance control.

From Figure 12, the charging current remains constant and the voltage increases slightly during the constant current stage. The voltage does not change and the current decreases rapidly during the constant voltage stage. In other words, by controlling the primary side impedance, the obtained battery characteristic curve is consistent with the battery characteristic curve under the direct control of the primary side, so it can be proved that the scheme of controlling the primary side impedance is feasible.

In order to charge the battery in different states, in the constant current stage, the primary side impedance is subjected to constant resistance control, that is, the reference impedance is set to a constant value, and the battery characteristic curve is obtained as shown in Figure 13.
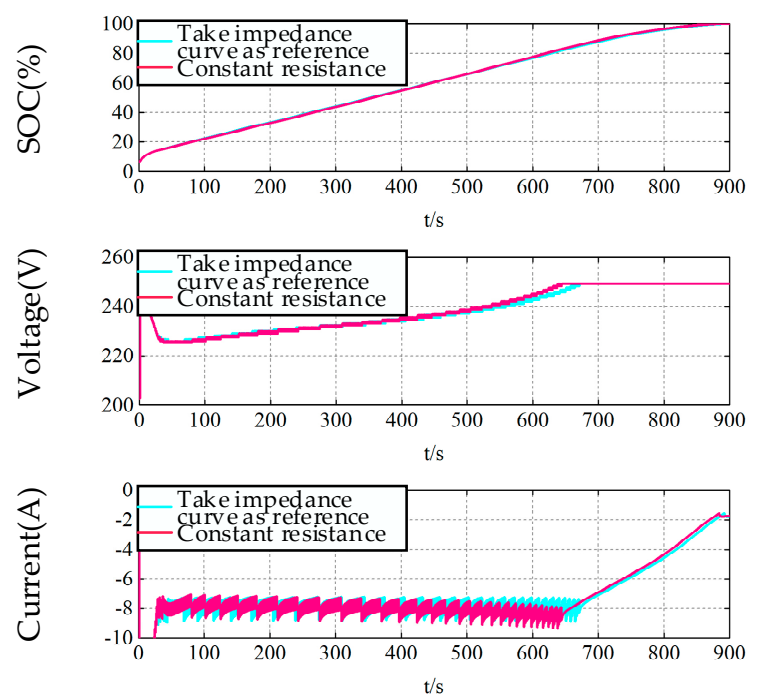

Figure 13. The characteristic curve of the primary side constant impedance control.

As it can be seen from Figure 13, the blue waveform is obtained by controlling the reference impedance curve, and the red one is obtained by the constant resistance, and they are substantially 
coincident. In the constant current charging stage, the battery voltage increases slowly. Because the primary side impedance is constant, the charging current will also increase slightly and the charging speed will also increase. By comparison, the results obtained by using the constant resistance control are basically consistent with those obtained by using the impedance reference curve. Therefore, the constant resistance control in the constant current stage can effectively solve the problem of different starting points of the battery charging.

\subsection{Experiment}

On the basis of the structure diagram of system shown in Figure 1 and the system parameters shown in Table 1, a wireless charging experimental device was built to verify the effectiveness of the primary side impedance control method.

The experimental system control module consists of an ARM (ARM, Cambridge, UK) and an FPGA chip (Altera, San Jose, CA, USA). In the charging process, ARM chips are mainly used for signal acquisition and processing. The primary side inverter voltage and current information are collected in the initial stage of charging and converted into impedance information. With this impedance value as a reference, the impedance information of the primary side collected in real time is compared with the reference value. The resulting error information is sent to the FPGA chip to adjust the driving signal according to the error. When the battery voltage reaches the limit, constant voltage control is performed, and the control charging voltage is kept constant until the charging is completed. The experimental device is shown in Figure 14.

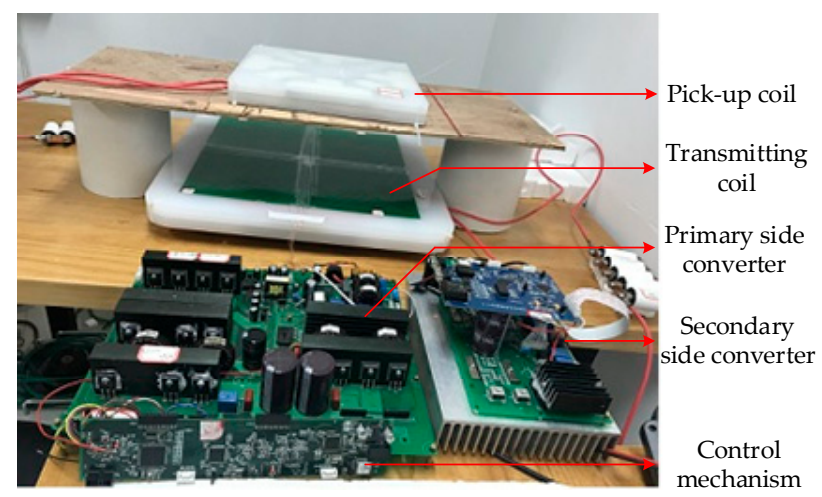

Figure 14. The diagram of experimental device.

The lithium ion battery is charged by controlling the primary side impedance. In the constant current charging stage, the voltage and current waveforms of the primary side are as shown in Figure 15.

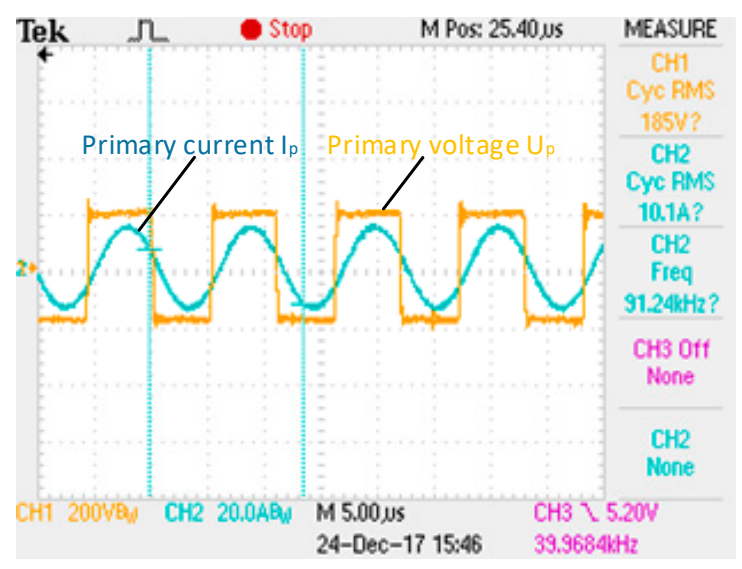

Figure 15. The voltage and current waveforms in the primary side at constant current stage. 
It can be seen that the charging system operates in a resonant state, and the output voltage and current waveform corresponding to the secondary side are shown in Figure 16.

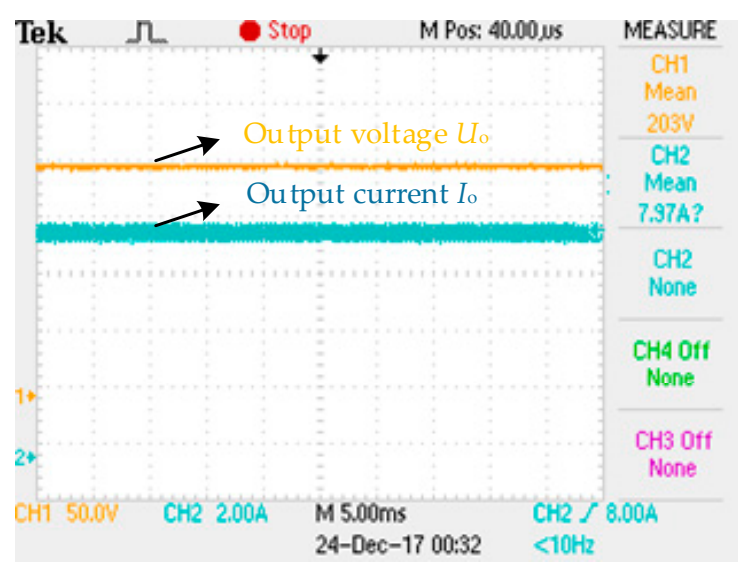

Figure 16. The output voltage and current waveforms in the secondary side at constant current stage.

It can be seen from Figure 16, through the control of the primary side impedance, the system realizes the constant current output, and the output current has a certain fluctuation $( \pm 0.2 \mathrm{~A})$, but the overall control effect is not affected. Due to the limited conditions, the experimental waveform cannot be viewed continuously, and only the test experimental waveform can be collected within a short time. Therefore, the output voltage is also constant, However, as the charging process progresses, the output voltage will rise slowly until the battery is the highest voltage.

In the constant voltage charging stage, the voltage and current waveform of the primary side are shown in Figure 17.

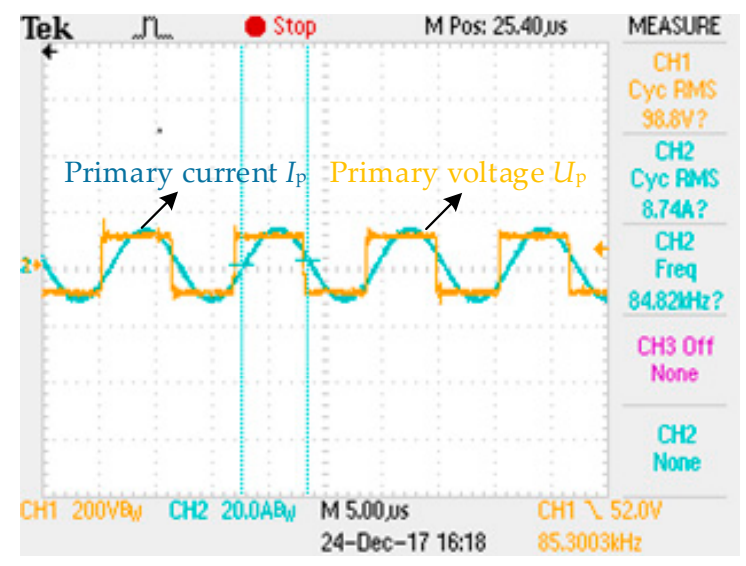

Figure 17. The voltage and current waveforms in the primary side at constant voltage stage.

Comparing Figure 17 with Figure 15, in the constant voltage stage, it can be seen that the battery power is close to saturation, so the power will be reduced at this time. Both the primary side voltage and current will be decreased. At this time, the output voltage and current waveform corresponding to the secondary side are shown in Figure 18. 


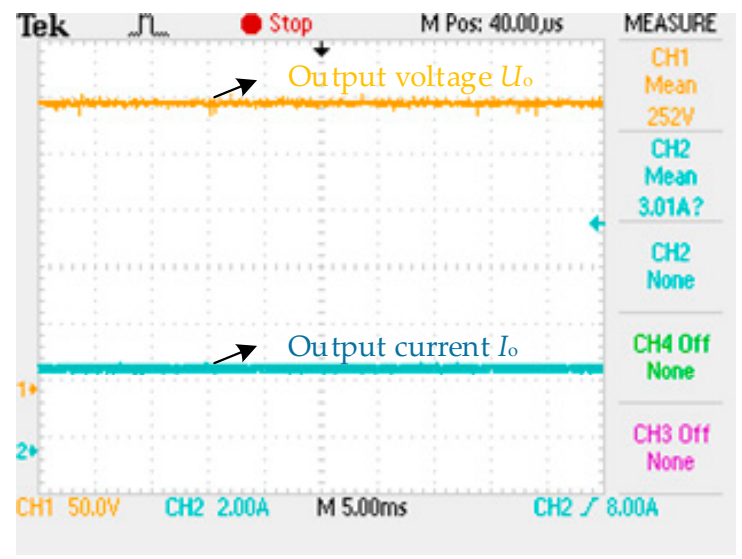

Figure 18. The output voltage and current waveforms in the primary side at constant voltage stage.

Comparing Figure 18 with Figure 16, in the constant voltage stage, it can be seen that the output voltage of the secondary side is increased while the output current of the secondary side is decreased, which is consistent with the previous theory.

The whole charging process was recorded, and the experimental data obtained are shown in Table 2. In the table, the measured values of constant current output and constant voltage output are marked in the brown area.

Table 2. Data of system.

\begin{tabular}{cccccccc}
\hline $\boldsymbol{Z}_{\boldsymbol{p}}(\boldsymbol{\Omega})$ & $\boldsymbol{U}_{\boldsymbol{p}}(\mathrm{V})$ & $\boldsymbol{I}_{\boldsymbol{p}}(\mathrm{A})$ & $\boldsymbol{U}_{\boldsymbol{o}}(\mathrm{V})$ & $\boldsymbol{I}_{\boldsymbol{o}}(\mathrm{A})$ & $\boldsymbol{R}_{\boldsymbol{e q}}(\boldsymbol{\Omega})$ & $\boldsymbol{P}_{\boldsymbol{o}}(\mathbf{k W})$ & $\eta(\%)$ \\
\hline 20.31 & 195 & 9.60 & 200 & 7.91 & 25.32 & 1.58 & 91.3 \\
20.20 & 200 & 9.90 & 210 & 7.90 & 26.58 & 1.66 & 91.6 \\
20.20 & 204 & 10.10 & 220 & 7.85 & 28.20 & 1.73 & 92.0 \\
20.19 & 208 & 10.30 & 230 & 7.85 & 29.20 & 1.81 & 92.3 \\
20.29 & 211 & 10.40 & 240 & 7.90 & 30.38 & 1.90 & 92.6 \\
20.47 & 216 & 10.55 & 250 & 7.90 & 31.65 & 1.98 & 92.8 \\
20.20 & 198 & 9.80 & 252 & 6.90 & 36.23 & 1.74 & 92.9 \\
20.18 & 181 & 8.97 & 251 & 5.80 & 43.10 & 1.46 & 92.5 \\
18.61 & 163 & 8.76 & 249 & 4.84 & 51.65 & 1.21 & 91.5 \\
14.81 & 129 & 8.71 & 248 & 3.85 & 64.94 & 0.95 & 90.0 \\
10.97 & 96 & 8.75 & 252 & 2.87 & 87.11 & 0.72 & 87.6 \\
\hline
\end{tabular}

It can be seen from Table 2 that by controlling the primary side impedance, the control of constant current charging and constant voltage charging can be completed, and the control precision is also high. However, due to the non-ideality of the structural parameters of the system and the errors caused by the readings in the test, the individual data are inconsistent with the theory, but the overall trend is consistent with the theory. The experimental data is plotted as a graph as shown in Figure 19.

It can be seen that the battery charging characteristic curve is consistent with the charging characteristic curve under direct control of the primary side in Figure 19. In other words, under the experimental conditions, by controlling the primary side impedance, the same effect as the direct control of the primary side can be achieved; through experimental verification, the scheme based on the primary side impedance control is effective. 

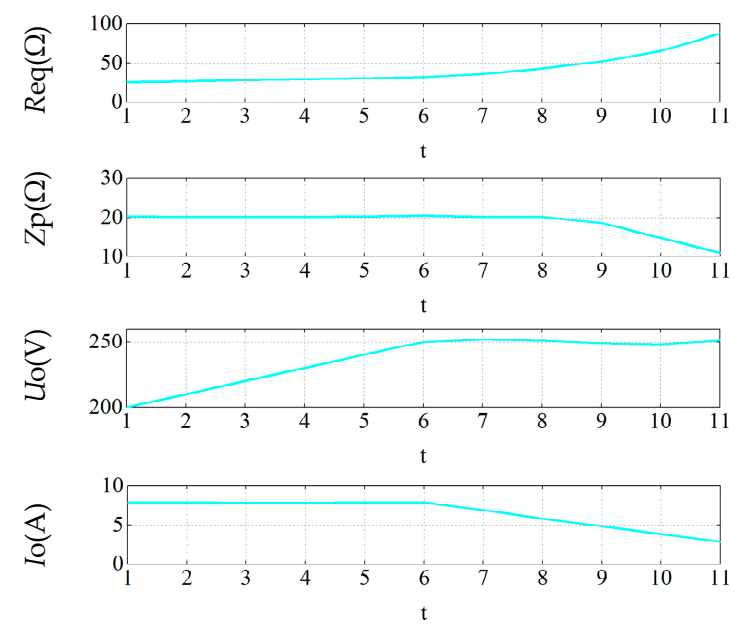

Figure 19. The experimental waveform diagram under the primary side impedance control scheme.

\section{Discussion}

Through the simulation and experimental analysis of the previous chapter, it is verified that the primary control method of a wireless charging system based on load characteristics proposed in this paper is achievable. Compared with the existing control methods, the proposed control method not only reduces the communication requirements of the system, but also simplifies the hardware circuit, reduces the complexity of the control circuit and reduces the volume and cost of the system. In addition, a solution is proposed to solve the problem of different initial quantity of battery, and the effectiveness of the solution is verified by simulation. However, there are still some shortcomings in this paper. By force of contrast, we can see that from Table 3, the control accuracy of the proposed method is not as high as that of the primary direct control and cooperative control method. So in the future, further research can be carried out from the angle of improving control accuracy.

Table 3. Method comparison.

\begin{tabular}{ccccc}
\hline Index & Proposed & Direct Control & $\begin{array}{c}\text { Cooperative } \\
\text { Control }\end{array}$ & $\begin{array}{c}\text { Indirect Control in } \\
\text { Reference [25,26] }\end{array}$ \\
\hline Communications & No need & Need & No need & No need \\
Circuit complexity & Simple & Medium & Complex & Complex \\
Control accuracy & Medium & High & High & Medium \\
Control complexity & Simple & Medium & Medium & Complex \\
Cost & Low & High & High & High \\
\hline
\end{tabular}

\section{Conclusions}

In this paper, a primary control method based on load characteristic identification is proposed to realize the accurate control of secondary output. Aiming at SS compensated Li ion wireless charging system, based on the load characteristic curve of the battery and AC impedance model, the mapping relationship between the secondary side output and the primary side impedance is established. Using this mapping relationship, the BUCK circuit is employed as the controller, and the output of the secondary side can be controlled by controlling the input voltage of the inverter only when there is no closed-loop control between the primary side and secondary side, as well as an additional circuit. Compared with the traditional control methods, the proposed method has the advantages of high precision, easy implementation, low cost, small size and easy popularization. The effectiveness and superiority of the control method are verified by MATLAB simulation analysis and experiments.

Author Contributions: Supervision, methodology, writing-original draft preparation, G.C.; software, formal analysis, C.R.; conceptualization, validation, investigation, resources, Y.S. and Z.C.; resources, data curation, C.T.; supervision, funding acquisition, Z.Z. 
Funding: National Natural Science Foundation of China (61573074); State Grid Corporation Science and Technology Project (5211DS17002F).

Conflicts of Interest: The authors declare no conflict of interest.

\section{References}

1. Wang, C.S.; Stielau, O.H.; Covic, G. Design considerations for a contactless electric vehicle battery charger. IEEE Trans. Ind. Electron. 2005, 52, 1308-1314. [CrossRef]

2. Zhang, X.; Jin, Y.; Yuan, C.; Wei, B.; Wang, S.; Zhao, M. Compact-Strong coupling model analysis of dynamic wireless charging for electric vehicles. Power Syst. Autom. 2017, 41, 79-83.

3. Covic, G.A.; Boys, J.T. Modern Trends in Inductive Power Transfer for Transportation Applications. IEEE Journal of emerging and selected topics in power electronics. 2013, 1, 28-41. [CrossRef]

4. Sun, Y.; Zhang, H.; Tao, W.; Ma, J.; Li, L.; Xia, J. Wide Load Constant Voltage Induction Coupled Power Transmission System Based on Variable Structure Mode. Power Syst. Autom. 2016, 40, 109-114, 126.

5. Sun, Y.; Xia, C.; Dai, X.; Su, Y. Analysis and optimization of mutual inductance coupling parameters of inductive coupling power transmission system. Chin. J. Electr. Mech. Eng. 2010, 30, 44-50.

6. Ahn, D.; Hong, S. Wireless Power Transmission With self-regulated Output Voltage for Biomedical Implant. IEEE Trans. Ind. Electron. 2013, 61, 2225-2235. [CrossRef]

7. Hu, C.; Sun, Y.; Tang, C.S.; Wang, Z.H. Optimal Design of Electromagnetic Coupling Mechanism for ICPT System. In Proceedings of the IEEE Conference on Industrial Electronics and Applications, Melbourne, Australia, 19-21 June 2013; pp. 1233-1237.

8. Sun, Y.; Dai, L.; Ye, Z.; Tang, C.; Tan, R. Reverse synchronous transmission technology of energy and signal in inductive coupling power transmission system. Power Syst. Autom. 2008, 42, 134-142.

9. Yang, M.; Wang, Y. Dynamic detuning transmission power control of inductive coupling power transmission system. J. Mot. Control 2012, 16, 72-78.

10. Hirai, J.; Kim, T.W.; Kawamura, A. Practical Study on Wireless Transmission of Power and Information for Autonomous Decentralized Manufacturing System. IEEE Trans. Ind. Electron. 1999, 46, 349-359. [CrossRef]

11. Huang, X.; Tan, L.; Chen, Z.; Qiang, H.; Zhou, Y.; Wang, W.; Cao, W. Research and application review of radio energy transmission technology. J. Electr. Technol. 2013, 28, 1-11.

12. Mascarenas, D.L.; Flynn, E.B.; Todd, M.D.; Overly, T.G.; Farinholt, K.M.; Park, G.; Farrar, C.R. Experimental studies of using wireless energy transmission for powering embedded sensor nodes. J. Sound Vib. 2010, 329, 2421-2433. [CrossRef]

13. Su, Y.-G.; Chen, L.; Tang, C.-S.; Ma, J. Hu, A.-S. Based on the NSGA-IIECPT system PID parameter optimization of the algorithm and the output voltage control. J. Electrotech. 2016, 31, 106-114.

14. Dai, X.; Li, X.; Li, Y.; Hu, A.P. Maximum Efficiency Tracking for Wireless Power Transfer Systems with Dynamic Coupling Coefficient Estimation. IEEE Trans. Power Electron. 2018, 33, 5005-5015. [CrossRef]

15. Xia, C.; Li, Y.; Lei, K.; Liu, Y.; Chen, G. Reverse synchronous transmission method of power and signal in variable load ICPT system. Chin. J. Electr. Eng. 2017, 37, 1857-1867.

16. Sun, Y.; Wang, Z.; Tang, C. Real-time synchronous transmission method of power signal sharing channel in ICPT system. Chin. J. Electr. Eng. 2016, 36, 5172-5178, 5398.

17. Zhou, J.; Sun, Y.; Su, Y.; Dai, X.; Zhai, Y. Induction coupling power and signal synchronous transmission technology. J. Chongqing Inst. Technol. 2009, 23, 93-97.

18. Hata, K.; Imura, T.; Hori, Y. Maximum efficiency control of wireless power transfer systems with Half Active Rectifier based on primary current measurement. In Proceedings of the 2017 IEEE 3rd International Future Energy Electronics Conference and ECCE Asia (IFEEC 2017_ECCE Asia), Kaohsiung, Taiwan, 3-7 June 2017; pp. 1-6.

19. Yang, M. Research on Contactless Inductive Coupling Power Transmission and Control Technology and Its Application. Ph.D. Thesis, Hunan University, Changsha, China, 2012.

20. Li, H.; Li, J.; Wang, K.; Chen, W.; Yang, X. A Maximum Efficiency Point Tracking Control Scheme for Wireless Power Transfer Systems Using Magnetic Resonant Coupling. IEEE Trans. Power Electron. 2015, 30, 3998-4008. [CrossRef] 
21. Yuan, L.; Li, B.; Zhang, Y.; He, F.; Chen, K.; Zhao, Z. Maximum efficiency point tracking of the wireless power transfer system for the battery charging in electric vehicles. In Proceedings of the 2015 18th International Conference on Electrical Machines and Systems (ICEMS), Pattaya, Thailand, 25-28 October 2016; pp. 1101-1107.

22. Su, Y.G.; Zhang, H.Y.; Wang, Z.H.; Hu, A.P.; Chen, L.; Sun, Y. The Steady-State Load Identification Method of Inductive Power Transfer System based on Switching Capacitors. IEEE Trans. Power Electron. 2015, 30, 6349-6355. [CrossRef]

23. Su, Y.; Chen, L.; Wang, Z.; Hu, A.; Dai, X. Load identification method of inductive power wireless transmission system based on least square method. J. Electrotech. 2015, 30, 9-14.

24. Su, Y.; Chen, L.; Wu, X.; Qing, X.; Tang, C. Load and mutual inductance identification method of SS magnetic coupling WPT system based on genetic algorithm. J. Electr. Technol. 2018, 33, 4199-4206.

25. Song, K.; Li, Z.; Du, Z.; Wei, G.; Zhu, C. Design for constant output voltage and current controllability of primary side controlled wireless power transfer system. In Proceedings of the 2017 IEEE PELS Workshop on Emerging Technologies: Wireless Power Transfer (WoW), Chongqing, China, 20-22 May 2017.

26. Zhai, Y.; Sun, Y.; Dai, X.; Su, Y.; Wang, Z. Parameter identification and primary side control of high intensity magnetic coupling resonance system. J. Mot. Control 2014, 18, 1-6.

27. Xu, L. Research on Power Lithium Battery Charging Technology. Master's Thesis, Taiyuan University of Science and Technology, Taiyuan, China, 2014.

28. Xiao, F. Research on Balanced Charging Technology of Lithium-Ion Battery Pack for Electric Vehicles. Master's Thesis, Tianjin University of Technology, Tianjin, China, 2011.

29. Su, Y.; Du, W.; Chen, Q.; Xia, Y. Rapid intelligent charging technology for lithium ion battery pack. J. Chongqing Inst. Technol. 2008, 1, 89-93.

(C) 2019 by the authors. Licensee MDPI, Basel, Switzerland. This article is an open access article distributed under the terms and conditions of the Creative Commons Attribution (CC BY) license (http://creativecommons.org/licenses/by/4.0/). 